\title{
Téoros
}

Revue de recherche en tourisme

\section{Musées et tourisme en France - 1970/1992}

\section{Valéry Patin}

Volume 11, numéro 2, juillet 1992

Quand les musées s’ouvrent au tourisme...

URI : https://id.erudit.org/iderudit/1078086ar

DOI : https://doi.org/10.7202/1078086ar

Aller au sommaire du numéro

Éditeur(s)

Université du Québec à Montréal

ISSN

0712-8657 (imprimé)

1923-2705 (numérique)

Découvrir la revue

Citer cet article

Patin, V. (1992). Musées et tourisme en France - 1970/1992. Téoros, 11(2), 13-15. https://doi.org/10.7202/1078086ar d'utilisation que vous pouvez consulter en ligne.

https://apropos.erudit.org/fr/usagers/politique-dutilisation/ 


\title{
Musées et tourisme en France - 1970/1992
}

\author{
Valéry Patin
}

En vingt ans, le nombre des musées comme celui des visiteurs ont fortementaugmenté. Lamuséographie, les thématiques proposés, la fonction économique des musces ont connu desmutations profondes. L'affirmationd'une politique culturelle ambitieuse, la relative dégradation de l'environnement économique, en particulier dans les zones rurales, ont très largement contribue a modifier les relations entre les lieux culturels et leurs publics. Les opérateurs culturels, élus ou techniciens, ont souvent considéré le tourisme comme une simple opportunité économique susceptible d'être développée par des opérations de valorisation du patrimoine, en ignorant fréquemment les spécificités de la pratique touristique en ce domaine. Spécificités liées à l'hétérogénéité des publics, des modalités de formation des goûts, aux contraintes de temps.

\section{Une offre culturelle en pleine mutation}

Durant les vingt demières annees, à Paris et en région parisienne, plusieurs muscées ont été créśs dont certains abritent des collections prestigieuses (Orsay, Musée Picasso). De grandes institutions ont été rénovées et agrandies (Musée du Louvre, Musée Carnavalet), d'autres le seront très prochainement (Musée de l'Homme, Palais de la Découverte, Galeries du Jardin des Plantes).

Depuis le succès de l'exposition Toutânkhamon qui, en 1967, avait accueilli plusd'un million de visites, les grandes manifestations temporaires rythment la vie culturelle et touristique de la capitale. La dernière en date, consacrée, au printemps 1992, à Toulouse-Lautrec a reçu plus de 650000 visites.

\footnotetext{
Monsieur Valéry Patin, docteur en sociologie, est directeur de la société Empreinte Communication SA (spécialisée dans la mise en valeur du patrimoine culturellet Maitre de Conférence associé à l'Université de Paris V. II est aussi conseil de Musée du Louvte pour la mise en place d une politique des publics touristiques.
}

En province, les créations et les rénovătions muséographiques ont été nombreuses également. Les musées des Beaux-Arts de Rouen ou de Lyon ouvrent au public de nouveaux espaces, apres des campagnes de travaux conséquentes. Dunkerque et Villeneuve d'Ascq, dans le Département du Nord, présentent d'exceptionnelles collections d'art moderne et contemporain provenant des donations et dations ${ }^{(1)}$ réalisées par les grandes familles textiles de la région. Le musée d'art moderne de Troyes en Champagne aété constitué également autour d'une donation du même type, la collection Levy. La ville de Marseille a rénové les locaux de l'ancienne Charité pour y abriter des expositions de très grande qualité. Un musée privé consacré à l'impressionnisme américain vient d'ouvrir ses portes à Giverny, à quelques mètres de la maison du peintre Claude Monet. De nombreux musées de sites, crés à proximité de vestiges historiques ou archéologiques, ont également êtế rếalisếs.

À Bélesta dans les Pyrćnćes-Orientales, tout près de Perpignan, un musée récent installé dans l'ancien château frontière, au centre d'un petit village viticole, présente au public une reconstitution de la plus ancienne scpulture néolithique du Midi, découverte à proximité. À Argentomagus, dans l'Indre, le musée s'inscrit directement dans le site romain dont il utilise la morphologie et les vestiges. À Senlis, dans une configuration similaire, le muscé intègre la muraille de I'enceinte romaine et se développe dans des bâtiments restaurés des XVII ${ }^{e}$ et XVIII ${ }^{e}$ siècles. Les collections, les témoignages architecturaux et plastiques sont ainsi présentes au public, in situ.

Plus modestes, en terme de fréquentation, mais d'un intérêt culturel essentiel, des écomusces ont vu le jourcesdernièresannées. A titre d'exemple, on peut citer le Centre Minier de Lewarde, dans le Nord, construit sur des puits de mines désaffectés que font visiter $\mathrm{d}^{\dagger}$ anciens mineurs $\mathrm{y}$ ayant travaillé lorsqu'ils étaient en exploitation. C'est également la réussite spectaculaire de l'écomusée d'Alsace à Mulhouse où l'on trouve rassemblés les témoignages de l'architecture vernaculaire alsacienne (architec- ture à pan de bois). Les réalisations sont nombreuses dans ce domaine, Musée du sel à Marsal en Lorraine, Musée de la pêche à Concarneau en Bretagne, Musée du papier à Ambert en Auvergne.

D'autres réalisations connaissent depuis peu de temps un succès considérable, ce sont les aquariums. D'une conception architecturale spectaculaire, abritant de multiples variétés animales et végétales, ces nouveaux lieux du tourismeaccueillent une fréquentation considérable (1,3 million de visites à Brest, 800000 à La Rochelle).

Une telle explosion de l'offre culturelle constitue l'élément le plus impressionnant d'une réalité marquée par.

* l'evolution des usages touristiques du patrimoine;

- la mise en oeuvred'une politique culturelle très ambitieuse;

- l'incidence d'une situation ćconomique dégradée, en particulier dans le milieu rural;

- l'évolution des mentalités professionnelles.

\section{L'évolution des usages touristiques du patrimoine culturel}

En quelques annees, les usages touristiques du patrimoine culturel se sont profondément modifiés.

En premier lieu, la fréquentation de nature strictement touristique, c'est-à-dire réalisćc àl'occasion d'un déplacement plus ou moins long de vacances ou d'affaires, a connu un taux de croissance considérable. En 1991, sur 5 millions de visites enregistrées au Louvre, 3 millions étaient le fait d'étrangers (dont 30000 Canadiens) en séjouren France, et 1 million de Français provinciaux en séjour dans la capitale ou en région parisienne. Les parisiens (Paris et région parisienne) représentaient moins de $20 \%$ du total des visiteurs.

En second lieu, et quels que soient les sites, une sćrie d'études a permis de constater ${ }^{(2)}$. 
- qu'en période de congés, les résidants permanents, non vacanciers, constituaient une clientèle importante pour les musées et monuments de leur région;

- qu'une proportion notable de visiteurs touristiques du patrimoine en période de vacances ne visitait jamais de sites culturels pendant le reste de l'année.

Les vacances constituent donc pour une majorité de visiteurs potentiels, résidants ou touristes, le moment privilégié de la consommation culturelle.

Enfin, l'anallyse régulière des comportements culturels des Français ${ }^{(3)}$, montre qu'au-delà du fait touristique, les visiteurs des musées, expositions, monuments, développent aujourd'hui une pratique beaucoup plus intensequ'auparavant. Le nombre des visiteurs non touristiques n'a pas beaucoupaugmente en vingt ans, mais le nombre des visites a, pour sa part, fortement progressé. Les visiteurs non touristigues sont donc des usagers réguliers des sites culturels, sensibles à la qualite des offres qui leur sont faites.

\section{La mise en oeuvre d'une politique culturelle ambitieuse}

Une telle mutation de l'offre muséographique et plus largement patrimoniale, est, au-delà du fait touristique, la conséquence d'une politique culturelle tout à fait exceptionnelle. Politique qui s'est traduite au niveau de l'administration centrale:

- par la mise en oeuvre d'une programmation financière conséquente, au regard, en tous cas, des moyens dont disposent habituellement les Affaires Culturelles;

- par la création d'un Secrétariat d'État chargé des Grands Travaux et dếpendant du Ministère de la Culture dirigé par monsieur J. Lang. Ce Secrétariat d'État a, par exemple, en charge les travaux du Grand Louvre budgétés pour un total de plus de 600 millions de francs;

- la transformation de la Réunion des Musées Nationaux (entité administrative responsable de la gestion des établissements et des collections appartenant a l'État) en établissement public à caractère industriel et commercial, ce qui autorise une plus grande souplesse dans la gestion commerciale, la gestion du personnel, de l'accueil.

Au niveau des régions et départements, la prise en compte de ces phénomènes culturels et touristiques a donné lieu à la création:

- de commissions spécialisées associant élus et techniciens;

- de postes de chargés de mission, d'animateurs du patrimoine;

- debudgétisations spéciliquesattribuées aux opérations de mise en valeur touristique du patrimoine culturel, financements répartis entre les communes, les départements, les régions, l'État et la Communauté Européenne, très présente dans ce domaine. $\mathrm{A}$ ce titre, cesactions s' inscriventaujourd' hui régulièrement dans les contrats quinquennaux conclus entre l'État et les Régions.

\section{L'incidence d'une situation économique dégradée, en particulier en milieu rural}

La situation de crise mondiale qui a prévalu durant plusieurs années a conduit les responsables à infléchir sensiblement leur conception du développement culturel. Le patrimoine s'est ainsi trouvé investi d'une vocation économique qu'il n'avait pas antérieurement, en toutcas, clairementaffirme $\mathrm{e}^{(4)}$.

Que ce soit au plan national avec la transformation de la Réunion des Musées Nationaux en Établissement Public Industriel et Commercial, que ce soit au plan local avec les multiples actions de mise en valeur économique du patrimoine (cf, par exemple les opérations menées par le Département de l'Aude sur les grands sites Cathares du sud de la France), les opcrateurs politiques et socioprofessionnels ont voulu orienter la gestion des musées, sites et monuments de telle manière que leur exploitation, tout en conservant leur caractère prioritairement culturel, crée des retombées économiques plus appréciables. Les travaux menés en ce domaine ont montré que le patrimoine culturel pouvait être créateur d'emplois et de richesses, en particulier par le biais du tourisme. En conséquence, lesmusées, siteset monuments ont vu leur rôle touristique s"accroitre très sensiblement au même titre que le balnéaire, la randonnée, les activités sportives et de détente. Dans une situation de chômage important (pratiquement $10 \%$ des actifs français sont au chômage), dans les zones rurales en voie de désertification, la création de 2 ou 3 emplois directs et induits pour 20 à 25000 visites payantes, une dépense moyenne par visiteur de $30 \mathrm{~F}$ à $40 \mathrm{~F}$ sur les sites et de $150 \mathrm{~F}$ a $200 \mathrm{~F}$ dans les hébergements et restaurants proches représentent parfois des éléments vitaux.

En contrepartie, cette nouvelle dimension économique acquise par le culturel a conduit à quelques excès. La réhabilitation de sites de peu d'intérêt historique ou artistique, la création de collectionsmuséographiques sans rapport avec l'identité culturelle régionale (par exemple un musée du Père Noèl dans une station balnéaire du LanguedocRoussillon), la promotion exclusive du patrimoine sans souci des autres éléments constitutifs de l'offre touristique (hébergement, sites naturels, équipements sportifs, etc.) amené certaines communesà des échecs financiers lourds de conséquence.

\section{L'évolution des mentalités et des pratiques professionnelles}

L'irruption du tourisme a contraint les responsables du patrimoine muséographique ou monumental à infléchir leurs usages professionnels à la fois pour communiquer différemment des contenus culturels à des publics souvent peu formés à la pratique des musées, monuments et autres sites, mais également à faire la part du feu en acceptant peu ou prou les nouvelles dimensions économiques de l’exploitation culturelle.

\section{Mieux communiquer le contenu culturel des musées et des sites patrimoniaux}

La tradition française en matière de muséographie et, plus largement, de présentation du patrimoine culturel au public a toujours privilégié une approche esthétique soutenue par un discours didactique instruit de référents culturels fédérateurs: l'histoire, en particulier l'histoire événementielle, la rationalité, la hiérarchie(s).

Les dimensions sociales, économiques, politiques de l'ocuvre d'art, quelle que soit sa nature, architecturale, plastique ou picturale, n'ont jamais constitué un système d'explication dominant. Cette pratique a conduit, 
jusqu'à une période récente, à la constitution d'un milieu cultivé, producteur ou reproducteur de la connaissance culturelle, et opérateur de sa diffusion auprès du grand public.

Elle aégalement conduità marginaliser voire exclure tous ceux qu' une telle approche rebutait soit parce qu'ils ne disposaient pas du code social nécessaire à sa compréhension, soit parce qu'un discours fédérateur, parfois univoque, ne leur paraissait plus apte à transmettre les multiples réalités du patrimoine.

Les recherches historiques récentes ${ }^{(6)}$, la présence de sensibilités économiques et sociales dans l'étude du patrimoine et l'utilisationde méthodologies issues de disciplines scientifiques - biologie, chimie, physique, médecine (par exemple la paléopathologie) ont fait évoluer très sensiblement la situation du système culturel traditionnel. Cette mutation s'est traduite par l'émergence de nouvelles modalités de présentation des oeuvres, par l'évolution des thématiques proposées, parune diversification dudiscours didactique.

Lamuséographiecherche, plus fréquemment, à créer autour de l'oeuvie ou du témoignage, un environnement émotionnel, sonore ou visuel, plus sensible qu'intellectuel (Mémorial de la Paix à Caen en Normandie). Les thêmatiques proposées font une plus large place aux approches sociologiques ou ethnologiques. Le musée de l'Homme a ainsi présenté des expositions importantes sur les rituels de la mort ou l'origine de l'homme.

Le discours de présentation du contenu culturel intè gre plus aisément des références à la vie quotidienne, à l'imaginaire, aux représentations sociales, aux structures administratives ou économiques. On voit se développer une pédagogie du patrimoine qui recourt à des instrumentations plus pratiques comme par exemple lesateliers ou les classes de patrimoine. Mais l'adaptation du discours culturel à différents types de publics, jeunes, adultes, retraités, issus de cultures différentes, reste encore un chantier pour les années à venir. Le concept d'interprétation ne fait pas partie des usages français avec une exception peut-être chez les spécialistes du milieu naturel, domaine dans lequel il existe en effet, mais en nombre limite, des Centres d'Interprétation de l'Environnement.
Accepter la dimension économique du patrimoine

La capacité du patrimoine à produire des richesses économiques, en particulier par le biais de l'activité touristique, semble aujourd'hui bénéficier d'un agrément majoritaire. En contrepartie, les conditions pratiques de cette valorisation économique sont appréciées de manière plus contrastée.

La présence de plus en plus fréquente de boutiques dans les musées, l'accroissement du nombre et des types d'objets, ouvrages, reproductions ${ }^{(7)}$ proposés a la vente, la progression des chiffres d'affaires ainsi engendrés constituent des phénomènes acceptês pour l'essentiel par les responsables culturcls.

La création de prestations induites à vocation touristique, tels les systèmes de réservation individuels et groupes ou les produits touristiques à forfait organisés autour de manifestations culturelles, en particulier, les expositions internationales ne semblent pas poser deproblèmeset sedéveloppent régulièrement.

Il n'en va pas de même lorsqu'il s'agit de créer autour du patrimoine culturel une véritable logique de croissance économique touristique, logique impliquant la réalisation d'infrastructures hôtelières et de restauration et, dans certains cas, la délégation de la gestion patrimoniale au secteur privé. Les tentatives menćes dans ce domaine depuis trois ou quatre ans par les groupes industriels comme par exemple le groupe ACCOR (Novotel) ne connaissent qu'un succès très relatif. La centralisation de l'administration culturelle, l'appréhensiondevant de possibles dérives d'une privatisation du patrimoine, le souci des gestionnaires privés de ne pas assurer les charges d'entretien et de restauration des collections et des monuments expliquent sans doute les résistances que l'on observe actuellement dans ce domaine.

\section{Conclusion}

L'accroissement de la fréquentation touristique des musées, monuments et sites a sans doute servi de révélateur dans un monde culturel naguère plus préoccupé de la sauvegarde des biens que de leur présentation au grand public. Mais la crise économique et l'affirmation d'une politique culturelle du patrimoine, en particulier muséographique, ont joué un rôle au moins aussi important.
L'État et les collectivités territoriales ont mis en oeuvre une politique de création et de rénovation muséographique et monumentale exceptionnelle. Les modalités de présentation des contenus culturels ont évolué. Les mises en valeur émotionnelles ont désormais leur place. On a vu se développer une pédagogie pratique de l'oeuvre culturelle grâce aux ateliers et classes de patrimoine. Le discours des guides et des conférenciers se réfere plus fréquemment à des systèmes d'explication socio-économiques ou scientifiques. Le patrimoine voit sa vocation à créer des richesses économiques mieux affirmées.

En cela le tourisme a exercé sur le monde culturel, en particulier celui des musées et monuments historiques, une influence notable. Il reste toutefois beaucoup à faire:

- chez les professionnels du tourisme à forfait pour qui l'objet culturel muséographique ou monumental a été totalement banalisé au même titre que la promenade en bateau-mouche ou la soirée au Lido;

- chez les professionnels de la culture pour qui les particularités de la consommation touristique du patrimoine avec ce que cela signifie - contraintes de temps, visite souvent unique, diversité des origines et des niveaux culturels demeurent encore l'objet d'interrogations et d'analyses, et n'ont pas donné lieu jusqu'à présentà l'élaborationd'une doctrine d'aménagement et de présentation des lieux culturels

\section{NOTES}

(1) Dation: système fiscal qui permet d'acquitter les droits de succession au moyen d'oeuvres d'art.

(2) Martin Garay, Le tourisme culturel en France, La Documentation Française, Paris, 1960

(3) Ministère de la Culture, Les pratiques culturelles des Français, La Documentation Française, Paris, 1990.

(4) Empreinte Communication, La valorisation touris. tique du patrimaine culturel, Développement Culturel, no 83, Paris, 1990

Xavier Greffe, La valeur bconomique du patrimoine, Anthropos, Paris, 1990.

(5) Pierre Francastel, Peinture et société, Paris; Gallimard, 1965

(6) Voir Fernand Braucel. L'identité de la France. Paris, Arhaud, 1986 ou lacques Le Goff, L'imaginaire mediéval, Paris, Gallimard, 1985.

(7) Le catalogue publié par la Réunion des Musées Nationaux propose plus de 2000 reproductions d'objets d'art (sculpture, bijoux, étoffes, gravures) appartenant pour la plupart aux collections de l'État. 